\title{
Energetics of Photosynthetic Algal Growth: Influence of Intermittent Illumination in Short (40 s) Cycles
}

\author{
By YUAN-KUN LEE* AND S. JOHN PIRT \\ Microbiology Department, Queen Elizabeth College (University of London), Campden Hill, \\ London $W 87 A H$
}

(Received 19 September 1980)

\begin{abstract}
A theory is developed to predict the growth rates of photosynthetic microbes in a photobiological reactor with light-sufficient zones and dark zones. The theory also predicts that in light-limited cultures the maintenance energy will increase in proportion to the duration of the dark period.

The theory was tested and quantitatively confirmed by means of a loop reactor which permitted light and dark periods to be varied with a total cycle time of $40 \mathrm{~s}$. An axenic Chlorella culture grown at the maximum rate under light-sufficient conditions could continue growth at the maximum rate in the dark for $9.2 \mathrm{~s}$. It was found that the maintenance energy was zero in the light, but endogenous metabolism of resting cells in the dark corresponded to a large maintenance energy of $8.8 \mathrm{~kJ}(\mathrm{~g} \text { dry wt) })^{-1} \mathrm{~h}^{-1}$. These results have much significance for the design of photobiological reactors.
\end{abstract}

\section{INTRODUCTION}

Light and dark zones in a photobiological reactor, with consequent intermittent energy supply to a photosynthetic culture in the reactor, may be unavoidable. Understanding the effects of intermittent illumination is therefore crucial to the design of photobiological reactors, which must be one of the main goals of microbiologists in the next decade in order to study cellular photosynthesis and to realize its great potential for harnessing solar energy.

Intermittent illumination of photosynthetic organisms is analogous to discontinuous feeding of a heterotrophic culture with its energy source. For instance, studies on methanol-oxidizing bacteria have shown that discontinuous feeding with methanol reduces the growth yield (Brooks \& Meers, 1973). If light as the sole energy source were cut off, two situations may be envisaged: one is that the organisms have a sufficient internal energy reserve to permit continuation of synthesis and cell growth; secondly, intracellular energy reserves could be exhausted so that growth must cease. If the latter is the case, endogenous metabolism of cell constituents could be stimulated, as pointed out by Pirt $(1975$, p. 70$)$ in his account of cell cultures at or near zero growth rate. Endogenous metabolism would result in a decrease in the biomass. The theoretical model advanced here is based on the biomass and energy balances of the processes.

Studies on photosynthetic metabolism have distinguished between the 'light reaction' and the 'dark reaction'. The 'light reaction' in green algal photosynthesis refers to the splitting of water to release $\mathrm{O}_{2}$, store reducing power and generate ATP; the 'dark reaction' refers to the fixation of $\mathrm{CO}_{2}$ and the synthesis of biomass using the reducing power and ATP generated in the light reaction. Attempts have been made to determine how long the dark reaction can continue after the cells have been illuminated by a light flash. Results in this field were summarized by Phillips \& Myers (1954). The flash times used were very short, ranging from 
0.01 to $67 \mathrm{~ms}$, and the dark reaction was observed to continue for up to $300 \mathrm{~ms}$. Livanský (1979) developed, by an extremely complex mathematical analysis, a model to account for the influence of dark zones in a culture on the overall growth rate, but he gave no experimental test of his model. His one prediction, which differs from ours, is referred to in the Discussion of the present paper. In contrast to the short, light-dark cycles, diurnal illumination is an example of a long cycle of intermittent illumination. Quite probably the diurnal light-dark cycle differs qualitatively from short cycles because it would be long enough to permit within the cycle substantial variations in the amounts of enzymes and other structural components in the cells, whereas, by definition, short cycles are not long enough to cause a substantial change in cellular composition during the cycle. The present paper is concerned with short light and dark periods (cycle time about $40 \mathrm{~s}$ ). Diurnal effects are beyond the scope of this paper.

For the experimental study of intermittent illumination effects, a novel technique was developed, based on the 'loop reactor', that is, a tubular reactor around which the culture is cycled. A part of the loop was blacked out to create a dark zone. The model and experimental technique developed allow the duration of the dark reaction in a growing photosynthetic culture to be determined definitively. The study also reveals important effects of intermittent illumination on the energetics of photosynthetic growth and the growth yields.

\section{THEOR Y}

\section{Biomass balance in light-sufficient cultures}

The loop reactor with culture recycle used for photosynthetic algal growth is represented diagrammatically in Fig. 1. The theoretical model of the intermittent light effects involves the following assumptions. The culture growth is not limited by nutrients in the medium. The culture flows turbulently along the tubular reactor. The cycle time in the loop is small enough to make the biomass concentration $(x)$ practically constant throughout the reactor, that is, the increase in biomass concentration in one cycle, $\Delta x \ll x$. The reactor has a total volume $V$; a fraction $\alpha$ of the total volume is illuminated. It is postulated that, as a result of storage of light energy by the organisms in the illuminated zone, $\mathrm{CO}_{2}$ fixation and growth may continue in the dark for a period. This 'dark growth zone' is designated as a fraction $\beta$ of the whole culture volume. The remainder of the dark zone in which growth is arrested (the 'dark rest zone') will be a fraction $\{1-(\alpha+\beta)\}$ of the culture volume. Also, it is assumed that growth restarts without lag when the organisms pass from the dark to the light zone.

Let the resting cells in the dark have a specific endogenous metabolic rate $a_{\mathrm{m}}$. This factor $a_{\mathrm{m}}$ can be equated with the 'specific maintenance rate' (Pirt, 1975) which is related to the notional maintenance coefficient $\left(m_{\mathrm{r}}\right)$ of the resting cells by the relation $a_{\mathrm{m}}=m_{\mathrm{r}} Y_{\mathrm{G}}$, where $Y_{\mathrm{G}}$ is the true growth yield from light (Pirt, 1975, p. 68).

The biomass balance for the loop reactor is

$$
\underset{\text { growth }}{\text { Net }}=\begin{aligned}
& \text { Growth in } \\
& \text { light zone }
\end{aligned}+\begin{aligned}
& \text { Growth in } \\
& \text { dark zone }
\end{aligned}-\frac{\text { Biomass decrease through endogenous }}{\text { metabolism in dark rest zone }}
$$

For a very small time interval $\Delta t$, this balance is

$$
\mu_{\mathrm{o}} x V \Delta t=\mu x \alpha V \Delta t+\mu x \beta V \Delta t-m_{\mathrm{r}} Y_{\mathrm{G}} x V\{1-(\alpha+\beta)\} \Delta t
$$

where $\mu_{\mathrm{o}}$ is the 'observed specific growth rate', which would be obtained from a plot of $x$ against time $(t)$, and $\mu$ is the specific growth rate in the growth zone (light or dark). From equation (1) we obtain

$$
\mu_{\mathrm{o}}=\mu(\alpha+\beta)-m_{\mathrm{r}} Y_{\mathrm{G}}\{1-(\alpha+\beta)\}
$$

The second term on the right-hand side of equation (2) represents the biomass loss due to the 


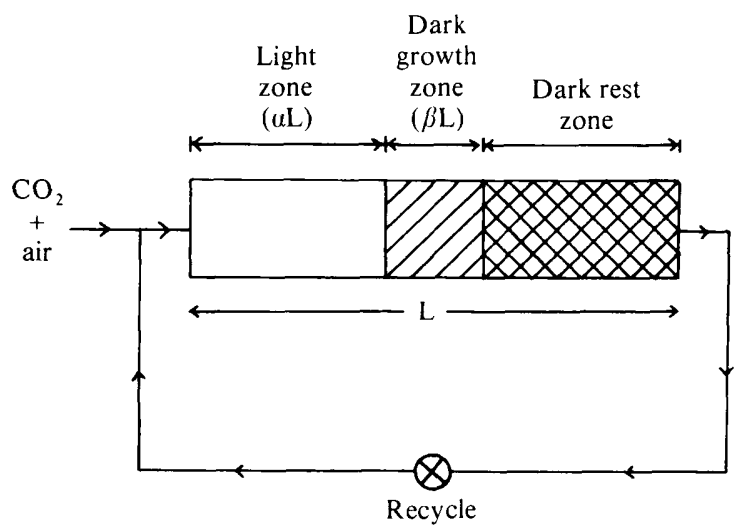

Fig. 1. Model of a loop reactor for photosynthetic algal growth.

endogenous metabolism of the resting cells. If this endogenous metabolism term is small compared with the growth, so that it can be neglected, and the specific growth rate $(\mu)$ has its maximum value $\left(\mu_{\mathrm{m}}\right)$, then equation (2) reduces to

$$
\mu_{\mathrm{o}} / \mu_{\mathrm{m}}=\alpha+\beta
$$

Experimentally the condition for equation (3) should be obtained if the culture is both energyand nutrient-sufficient in the growth zones. Thus the presence of a dark growth zone can be tested for by a plot of $\mu_{\mathrm{o}} / \mu_{\mathrm{m}}$ against $\alpha$. This plot should be a straight line with an intercept $\beta$ on the ordinate when $\alpha=0$.

\section{Energetics of light-limited growth}

Let the incident light intensity be $I_{0}$, the fraction of photosynthetically available radiation be $\phi$, the total surface area of the culture be $\mathrm{A}$, and the total culture volume be $V$, and other symbols as before. The energy balance will be

$$
\underset{\text { absorbed }}{\text { Light energy }}=\underset{\text { for growth }}{\text { Energy required }}+\begin{gathered}
\text { Energy required } \\
\text { for maintenance }
\end{gathered}
$$

where the term 'maintenance' covers functions other than growth. With light-limited growth it is assumed that all the photosynthetically available radiation incident on the culture is absorbed so that the energy balance becomes

$$
\phi I_{0} \mathrm{~A}=\mu_{\mathbf{0}} x V / Y_{\mathbf{G}}+m x V
$$

hence,

$$
\phi I_{0} \mathrm{~A} / x V=\mu_{\mathrm{o}} / Y_{\mathrm{G}}+m
$$

where the term on the left-hand side is the specific light absorption rate $(q)$. So far it has been assumed that maintenance energy is consumed at a constant specific rate $(m)$ throughout the growth and rest zones. However, whereas the light source can supply the maintenance energy required in the illuminated zone, endogenous metabolism must be the source of maintenance energy in the dark rest zone. The maintenance energies of the growing and resting cells may not be equal. Indeed, the evidence of studies on microbial behaviour at zero or near zero growth rate (Pirt, 1975, p. 70) shows that turnover of protein and degradation of cell constituents is stimulated when growth ceases. With the postulate that the maintenance energy in the growth zone is insignificant compared with that in the dark rest zone $\left(m_{\mathrm{r}}\right)$ the energy balance becomes

$$
\phi I_{0} \mathrm{~A}=\mu_{\mathrm{o}} x V / Y_{\mathrm{G}}+m_{\mathrm{r}} x V\{1-(\alpha+\beta)\}
$$


Table 1. Symbols, definitions and units

Symbol

Definition

Unit

A total surface area of culture

a specific maintenance rate

$a_{\mathrm{m}} \quad$ specific endogenous metabolic rate

$I_{0} \quad$ incident light intensity

$I_{\mathrm{T}} \quad$ transmitted light intensity

$m_{\mathrm{r}} \quad$ maintenance coefficient of resting cells

$m^{\prime}$ apparent maintenance coefficient of culture

specific light absorption rate

time

a small time interval

total culture volume

biomass concentration

increase in biomass concentration

true growth yield from light

actual growth yield

light zone, a fraction of the total volume illuminated

dark growth zone, a fraction of the total culture volume where cells continue

to grow in dark

$\delta$ dark rest zone, a fraction of the total culture volume where cell growth is arrested

$\mu \quad$ specific growth rate in the growth zone (light or dark)

$\mu_{\mathrm{m}} \quad$ maximum specific growth rate

$\mu_{\mathrm{o}} \quad$ observed specific growth rate

$\phi \quad$ fraction of photosynthetically available radiation

$\mathrm{m}^{2}$

$\mathrm{h}^{-1}$

$\mathrm{h}^{-1}$

$\mathrm{W} \mathrm{m} \mathrm{m}^{-2}$

$\mathrm{W} \mathrm{m^{-2 }}$

$J(g \text { dry } w t)^{-1} h^{-1}$

$J(\text { g dry wt })^{-1} h^{-1}$

$\mathrm{J}(\mathrm{g} \text { dry } w \mathrm{t})^{-1} \mathrm{~h}^{-1}$

$\mathrm{h}$ or $\mathrm{s}$

$\mathrm{h}$ or $\mathrm{s}$

$\mathrm{m}^{3}$

g dry wt $1^{-1}$

g dry wt $1^{-1}$

g dry wt $\mathrm{J}^{-1}$

g dry wt J-1

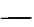

-
$\mathrm{h}^{-1}$
$\mathrm{~h}^{-}$
$\mathrm{h}^{-}$

hence,

$$
\begin{gathered}
\phi I_{0} \mathrm{~A} / x V=\mu_{\mathrm{o}} / Y_{\mathrm{G}}+m^{\prime} \\
m^{\prime}=m_{\mathrm{r}}\{1-(\alpha+\beta)\}
\end{gathered}
$$

where

Another postulate made is that when growth is light-limited in the illuminated zone, there is no storage of energy in the cells, so that growth cannot continue in the dark zone, that is, $\beta=$ 0 , hence equation (8) simplifies to

$$
m^{\prime}=m_{\mathrm{r}}-\alpha m_{\mathrm{r}}
$$

or, substituting $\alpha=1-\delta$, where $\delta$ is the dark fraction of the culture we obtain

$$
m^{\prime}=\delta m_{\mathrm{r}}
$$

Relation (10) can be tested experimentally by determining $m^{\prime}$ as a function of $\delta$.

Another possibility is that the maintenance coefficient $(m)$ could vary with the specific growth rate. An expression suggested by Neijssel \& Tempest (1976) for such an effect in heterotrophic growth is $m=m_{0}(1+k \mu)$, where $k$ is a constant, positive or negative according to whether $m$ increases or decreases with $\mu$. Such an effect would lead to variation in both the $m^{\prime}$ and the $Y_{\mathrm{G}}$ factors of equation (7). The complexities of such an effect are beyond the scope of this analysis.

Experimental tests of these theoretical models are described below. The definitions and units of the various symbols used are listed in Table 1.

\section{METHODS}

Cultures. Two algal cultures were used: one was a pure culture of Chlorella vulgaris 211/8k (Sorokin strain) obtained from the Culture Centre of Algae and Protozoa, Cambridge; the second was a defined consortium MA003 which consisted of a Chlorella-like alga A003 and three heterotrophic bacterial species (Pirt et al., 1980). 


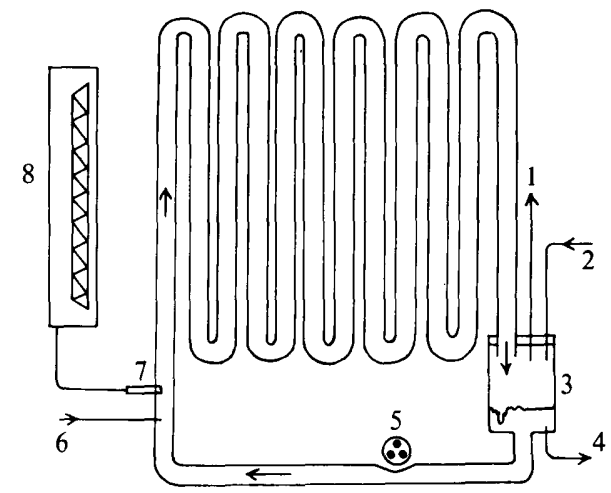

Fig. 2. Diagrammatic representation of the loop reactor: (1) gas outlet and condenser; (2) nutrient input; (3) degasser; (4) sampling point; (5) peristaltic pump; (6) $\mathrm{CO}_{2}$ input; (7) temperature sensor; (8) temperature controller and fan heater. The arrows indicate the direction of flow.

The cultures were grown photosynthetically on slopes of medium A9 plus agar $(2 \%, \mathrm{w} / \mathrm{v})$ under $5 \% \mathrm{CO}_{2}$ in air at $37{ }^{\circ} \mathrm{C}$. The stock cultures were stored at ambient temperature $\left(20-30^{\circ} \mathrm{C}\right)$ in the light.

Culture medium. Chemically defined medium $\mathrm{A} 9$ had the following composition (amounts in $\mathrm{g}^{-1}$ ): $\mathrm{KH}_{2} \mathrm{PO}_{4}$, 2.63; $\mathrm{CaCl}_{2} .2 \mathrm{H}_{2} \mathrm{O}, 0.08 ; \mathrm{MgSO}_{4} \cdot 7 \mathrm{H}_{2} \mathrm{O}, 3 \cdot 0 ; \mathrm{FeCl}_{3} .6 \mathrm{H}_{2} \mathrm{O}, 0.096 ; \mathrm{MnSO}_{4} \cdot 4 \mathrm{H}_{2} \mathrm{O}, 0.016 ; \mathrm{ZnSO}_{4} \cdot 7 \mathrm{H}_{2} \mathrm{O}, 0.018$; $\mathrm{CuSO}_{4} .5 \mathrm{H}_{2} \mathrm{O}, 0.004 ; \mathrm{Na}_{2} \mathrm{~B}_{4} \mathrm{O}_{7} .10 \mathrm{H}_{2} \mathrm{O}, 0.035 ; \mathrm{NaVO}_{3}, 0.005 ; \mathrm{NiSO}_{4} .7 \mathrm{H}_{2} \mathrm{O}, 0.0008 ; \mathrm{Na}_{2} \mathrm{EDTA}_{2}$ 2 $\mathrm{H}_{2} \mathrm{O}, 0.313$; urea, 4.0. The $\mathrm{pH}$ was $6 \cdot 7$. The medium was made up in four separate parts: (a) $\mathrm{KH}_{2} \mathrm{PO}_{4}$ adjusted to $\mathrm{pH} 6.7$ with $\mathrm{NaOH}$, (b) $\mathrm{CaCl}_{2}$ and $\mathrm{MgSO}_{4}$, (c) urea, and (d) other salts plus EDTA adjusted to pH 6.7 with $\mathrm{NaOH}$. Each stock solution was prepared and autoclaved separately at $121^{\circ} \mathrm{C}$ for $15 \mathrm{~min}$ and then mixed with the others after cooling.

The loop reactor. The tubular loop reactor is depicted in Fig. 2. It consisted of 12 Pyrex tubes, each about 25 $\mathrm{cm}$ long and of $0.7 \mathrm{~cm}$ bore, connected by Pyrex and silicone rubber U-bends. The tubing was laid horizontally and the end was connected to a vertical tube $(2.9 \mathrm{~cm}$ bore) which acted as a degasser. The culture volume was about $280 \mathrm{ml}$; this was checked when necessary with a measuring cylinder. The culture was recycled by means of a peristaltic pump (MHRE 200; Watson Marlow, Falmouth, Cornwall). The recycle rate was $420 \mathrm{ml} \mathrm{min}^{-1}$, which gave rise to a Reynolds number of 1000 , that is, about the lower limit for turbulent flow (Brown, 1961). The cycle time was $40 \mathrm{~s} . \mathrm{CO}_{2}\left(5 \%\right.$ in air) was injected at $15 \mathrm{ml} \mathrm{min}^{-1}$ into the reactor through a hypodermic needle installed downstream from the pump. The temperature of the culture was maintained at $37^{\circ} \mathrm{C}$ by means of a fan heater operated by a temperature controller (Pirt et al., 1979). The culture maintained a pH of $6 \cdot 5 \pm 0 \cdot 1$.

Illumination was provided by four horizontal warm white fluorescent tubes ( $20 \mathrm{~W}$, Philips) placed below the reactor tubes and parallel to them. The average light intensity at the surface of the reactor was $11 \mathrm{~W} \mathrm{~m}^{-2}$. The tubular reactor was placed under a white polyfoam cover to facilitate both maximum light input to the culture and the temperature control. When necessary, some of the tubes were covered by thick black paper to produce a dark zone in the reactor. The illuminated area was taken to be the entire inner surface of the tubes, that is, $2 \pi r l$ where $r$ is the inner radius and $l$ the length.

Adherence of cells to the walls of the tubular reactor (wall growth) did not occur under the conditions used.

Analytical methods. The turbidity $(A)$ of the culture was measured in a Unicam SP500 spectrophotometer at a wavelength of $680 \mathrm{~nm}$ with a $1 \mathrm{~cm}$ light path. At this wavelength the light absorption of the algal cells was maximal.

The specific growth rate $(\mu)$ and maximum specific growth rate $\left(\mu_{\mathrm{m}}\right)$ of the batch culture (Pirt, 1975, p. 22) of algae in the loop reactor were determined by measuring the increase in turbidity $\left(A_{680}\right)$ at short time intervals in light-limited and light-sufficient culture. The contribution of the bacteria to the turbidity was negligible at $680 \mathrm{~nm}$.

The algal biomass $(x)$ was estimated from the culture turbidity $\left(A_{680}\right)$. The ratio of turbidity to algal biomass remained fairly constant up to $40 \mathrm{~g}$ biomass $1^{-1}$ at all growth rates tested $\left(0.02-0.235 \mathrm{~h}^{-1}\right)$. An $A_{680}$ unit corresponded to $0.092 \mathrm{~g}$ dry biomass $\mathrm{l}^{-1}$ for Chlorella $211 / 8 \mathrm{k}$, and $0.124 \mathrm{~g}$ dry biomass $\mathrm{I}^{-1}$ for consortium MA003.

\section{RESULTS}

\section{Light-sufficient growth}

For growth to be light-sufficient the available light energy must exceed the maximum light required for growth, that is, $\phi I_{0} \mathrm{~A}>\mu_{\mathrm{m}} x V / Y$, where $Y$ is the actual growth yield. 


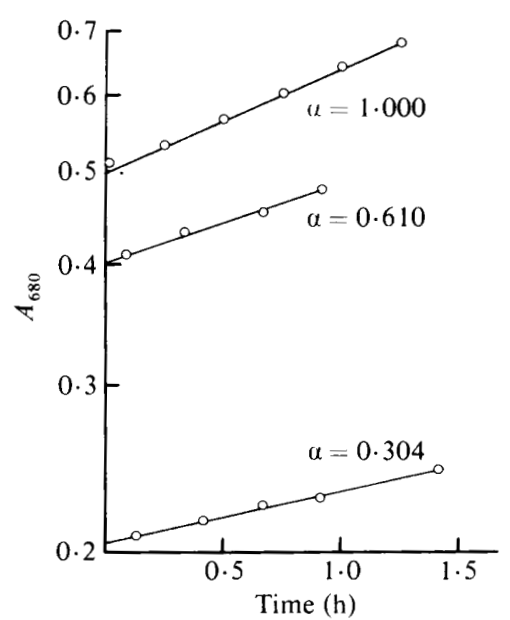

Fig. 3

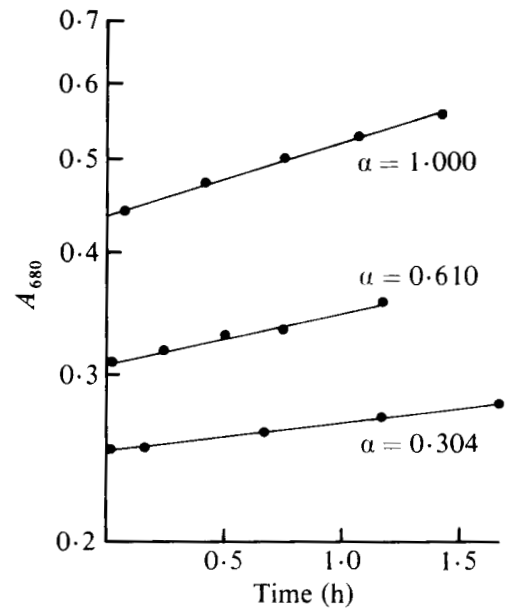

Fig. 4

Fig. 3. Exponential growth curves of light-sufficient batch cultures of Chlorella $211 / 8 \mathrm{k}$ at various light fractions $(\alpha)$.

Fig. 4. Exponential growth curves of light-sufficient batch cultures of consortium MA003 at various light fractions $(\alpha)$.

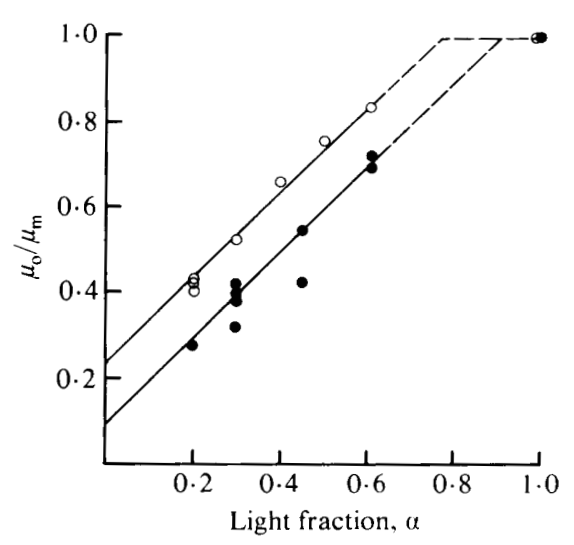

Fig. 5

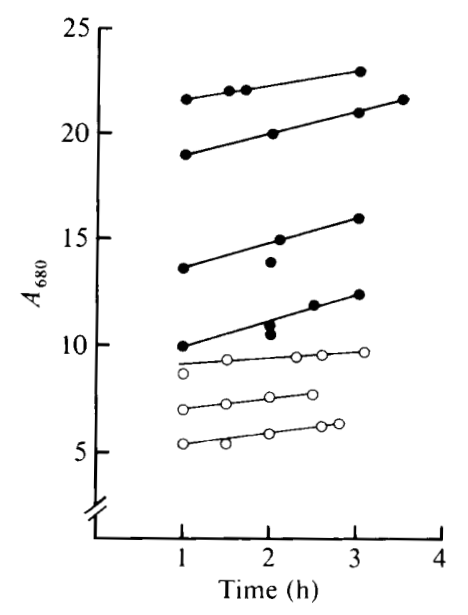

Fig. 6

Fig. 5. Plots of the ratio of the observed specific growth rate to the maximum specific growth rate $\left(\mu_{\mathrm{o}} / \mu_{\mathrm{m}}\right)$ as a function of the light fraction $(\alpha)$ for Chlorella $211 / 8 \mathrm{k}(\mathrm{O})$ and consortium MA003 $(O)$ in batch culture.

Fig. 6. Examples of the linear increase in turbidity $\left(A_{680}\right)$ with time for light-limited batch cultures of Chlorella $211 / 8 \mathrm{k}$ at $\alpha=0.405(\mathrm{O})$ and consortium MA003 at $\alpha=0.6$ (O). Each curve is for a different biomass concentration.

Accordingly, cultures were set up in the loop reactor with the aim of satisfying the above condition for light-sufficient growth. The growth curves of some of these cultures are shown in Figs 3 and 4 . When the cultures were illuminated along their entire length $(\alpha=1)$ the maximum specific growth rate of Chlorella $211 / 8 \mathrm{k}$ was $0.235 \mathrm{~h}^{-1}$ and of consortium MA003, $0 \cdot 170 \mathrm{~h}^{-1}$. To test equation (3) a dark zone was introduced along part of the reactor length. The ratio of the observed specific growth rate $\left(\mu_{\mathrm{o}}\right)$ to the maximum value $\left(\mu_{\mathrm{m}}\right)$ is plotted against the light fraction ( $\alpha$ ) for cultures of Chlorella $211 / 8 \mathrm{k}$ in Fig. 5. These data show that $\mu_{\mathrm{o}} / \mu_{\mathrm{m}}=1$ until the light fraction $(\alpha)$ was less than 0.77 . At lower $\alpha$ values $\mu_{\mathrm{o}} / \mu_{\mathrm{m}}$ 


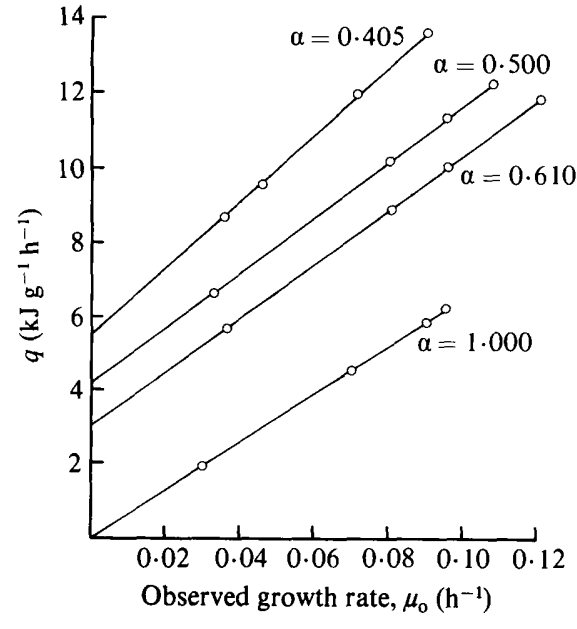

Fig. 7

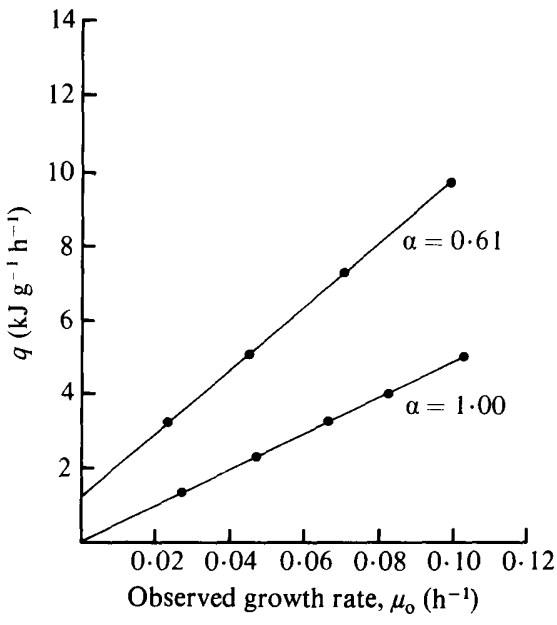

Fig. 8

Fig. 7. Specific light absorption rate $(q)$ as a function of the observed growth rate $\left(\mu_{0}\right)$ in light-limited batch cultures of Chlorella $211 / 8 \mathrm{k}$.

Fig. 8. Specific light absorption rate $(q)$ as a function of the observed growth rate $\left(\mu_{\mathrm{o}}\right)$ in light-limited batch cultures of consortium MA003.

was a linear function of $\alpha$ as predicted by the model in equation (3). The intercept on the ordinate gives $\beta=0.23$, that is, the maximum fraction of the culture in which 'dark growth' could occur. Since the cycle time in the loop reactor was $40 \mathrm{~s}$, the maximum duration of growth in the dark is deduced to be $0.23 \times 40=9.2 \mathrm{~s}$. When the light fraction $(\alpha)$ exceeded $0.77, \mu_{0}$ was equal to $\mu_{\mathrm{m}}$, which indicated that growth then extended to the full length of the culture system.

The plot of $\mu_{\mathrm{o}} / \mu_{\mathrm{m}}$ against $\alpha$ for consortium MA003 is also shown in Fig. 5. Again the plot was linear in accordance with equation (3). The value of the intercept $\beta$ was 0.09 ; thus the duration of the dark growth was $0.09 \times 40=3.6 \mathrm{~s}$.

\section{Light-limited growth}

Light-limited cultures were set up. The onset of light-limited growth is shown by the linearity of the increase in turbidity with time. However, if there is a finite maintenance energy, the rate of increase in turbidity will decrease with increasing biomass concentration. This is illustrated by the light-limited growth curves shown in Fig. 6. The observed specific growth rate of the culture $\left(\mu_{\mathrm{o}}\right)$ was calculated at different biomass concentrations $(x)$ from the ratio of $\sigma / x$, where $\sigma$ was the slope of the light-limited growth curve (Fig. 6). Plots of the specific light absorption rates $\left(q=\phi I_{0} \mathrm{~A} / x V\right)$ against the observed specific growth rates $\left(\mu_{0}\right)$ with different light fractions $(\alpha)$ are given in Figs 7 and 8. Both the axenic Chlorella $211 / 8 \mathrm{k}$ (Fig. 7) and the consortium MA003 (Fig. 8) showed a linear relation of $q$ and $\mu_{\mathrm{o}}$ for each $\alpha$ The slope of the plot is $1 / Y_{\mathrm{G}}$ and the intercept on the ordinate gives the value of the apparent maintenance energy coefficient which is $m^{\prime}$ according to the model equation (7).

For Chlorella 211/8k (Fig. 7) with the light fraction $(\alpha)$ above 0.5 the value of $Y_{\mathrm{G}}$ was constant and independent of $\alpha$. The mean and $95 \%$ confidence limits of $Y_{G}$ were $1.54 \times 10^{-5}$ $\pm 0.15 \times 10^{-5} \mathrm{~g}$ dry $\mathrm{wt} \mathrm{J}^{-1}$. The value of $Y_{\mathrm{G}}$ decreased significantly only when the light fraction was decreased to $0 \cdot 405$. One possible explanation is that when the light fraction of a culture decreases below a critical value for a particular photosynthetic organism, the maintenance energy or endogenous metabolism become dependent on both the light fraction and the specific growth rate. 


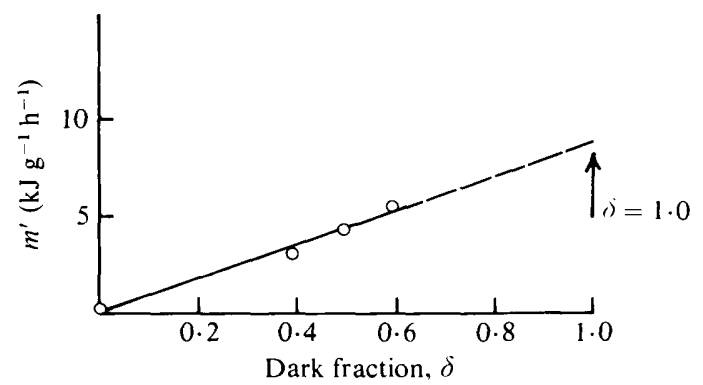

Fig. 9. Apparent maintenance coefficient $\left(m^{\prime}\right)$ as a function of the dark fraction $(\delta)$ in light-limited batch cultures of Chlorella $211 / 8 \mathrm{k}$.

The model equation (10) showing the effect of the light fraction on the maintenance coefficient $\left(m^{\prime}\right)$ was tested by plotting $m^{\prime}$ against the dark fraction $(\delta)$ as shown in Fig. 9 . The plot for Chlorella $211 / 8 \mathrm{k}$ shows that when $\delta=0$, the value of $m^{\prime}$ is practically zero, which is in agreement with the model postulates that for light-limited growth there is no growth in the dark zone $(\beta=0)$ and that the maintenance energy or endogenous metabolism is significant only in the dark zone. The maximum value of the maintenance coefficient $\left(m^{\prime}\right)$ was $8.8 \mathrm{~kJ}$ (g dry wt) $)^{-1} \mathrm{~h}^{-1}$.

With light-limited growth of the consortium MA003, increase in the dark fraction from 0 to 0.39 was associated with an increase in the slope of the $q$ versus $\mu_{\mathrm{o}}$ plot (Fig. 8) which means that there was a large reduction in the apparent maximum growth yield $\left(Y_{\mathrm{G}}\right)$, from $2 \cdot 1 \times$ $10^{-5}$ to $1.15 \times 10^{-5} \mathrm{~g}$ dry wt $\mathrm{J}^{-1}$. Also, Fig. 8 shows that the intercept on the ordinate, which corresponds to the maintenance coefficient, increased with increase in the dark fraction. The variation in both $Y_{\mathrm{G}}$ and the maintenance coefficient for the consortium MA003 could mean that the maintenance term depended on both the specific growth rate and the light fraction.

\section{DISCUSSION}

The loop reactor with light and dark zones is a novel means for the study of intermittent illumination in a photosynthetic culture. Previous workers on the problem, e.g. Kok (1953) and Phillips \& Myers (1954), used instead a flashing light, which seems less convenient for cycles of several seconds or longer duration. The new theoretical model of intermittent light effects proposed here has the virtues of simplicity and that it predicts important effects which can be tested experimentally. This model and the experimental technique have, for the first time, permitted definitive measurement of the duration of the dark growth reaction when the cultures are light-sufficient.

For Chlorella $211 / 8 \mathrm{k}$ the duration of the dark growth was $9.2 \mathrm{~s}$ when the specific growth rate was at its maximum value $\left(\mu_{\mathrm{m}}=0.235 \mathrm{~h}^{-1}\right)$. Hence, there must have been enough energy stored in the light-sufficient period to permit the biomass to increase by the fraction $\mu_{\mathrm{m}} \Delta t$ where $\Delta t$ is the dark growth period; this fraction was $6.0 \times 10^{-4}$ for Chlorella $211 / 8 \mathrm{k}$. It would be of interest to know the nature of the stored energy and reducing power which permit the continuation of biomass synthesis from $\mathrm{CO}_{2}$ in the dark; reduced NADP, ATP and a proton-motive force could be among the products involved.

Whereas we deduced that $\mu_{\mathrm{o}} / \mu_{\mathrm{m}}=\alpha+\beta$ (equation 3), and verified this experimentally for light-sufficient cultures, the theoretical model of Livansky (1979) led to the conclusion, in our notation, that $\mu_{\mathrm{o}} / \mu_{\mathrm{m}}$ approximately equals $\alpha^{2}$, provided $\alpha$ is not much less than unity. Livansky's model was not verified experimentally. Also, his model did not take account of the duration of the dark reaction or the effects on the growth yields.

Light-limited growth is important because it permits maximum utilization of the light source. However, light-limited growth will cause reduction of the specific growth rate; under 
this condition the maintenance energy requirement could cause a significant loss of energy. An important prediction of the model for the light-limited condition is that the maintenance energy will increase in proportion to the dark fraction of the culture. This prediction was quantitatively verified by the experimental measurements for light-limited cultures of Chlorella $211 / 8 \mathrm{k}$. Its maintenance coefficient was zero in the light and rose to $8.8 \times 10^{3} \mathrm{~J}(\mathrm{~g}$ dry wt $)^{-1} \mathrm{~h}^{-1}$ for resting cells in the dark. If the maintenance coefficient is expressed as a specific maintenance rate $\left(a=m^{\prime} Y_{\mathrm{G}}\right)$, the maximum value obtained for Chlorella $211 / 8 \mathrm{k}$ (when $\alpha=0$ ) is $8.8 \times 10^{3} \times 1.54 \times 10^{-5} \mathrm{~h}^{-1}$, that is, $0.136 \mathrm{~h}^{-1}$, which is more than half the maximum specific growth rate. Thus the endogenous metabolism rate in the dark zone was extremely high.

The dark response of the consortium MA003 was qualitatively similar to that of the axenic Chlorella 211/8k; however, the data, which are fewer, indicated that both $Y_{\mathrm{G}}$ and the maintenance energy were affected at a higher light fraction than was the case with Chlorella $211 / 8 \mathrm{k}$.

These findings have an important implication for deep algal cultures in which the light penetration is relatively small. The turbidity of the culture is given by $\log \left(I_{0} / I_{\mathrm{T}}\right)$, were $I_{0}$ is the incident light intensity and $I_{\mathrm{T}}$ is the transmitted light intensity. If the turbidity were 10 for a culture $1 \mathrm{~cm}$ deep, this means that $99 \%$ of the incident light would be absorbed or scattered in the $10^{-2} \mathrm{~cm}$ layer of culture next to the tube wall exposed to the light. This means that $97 \%$ of the culture would be effectively shaded by the outer $3 \%$ of the culture, assuming that the tube is illuminated over half its surface. Therefore, if the culture were light-limited, it should be imperative for the cells to move periodically into the thin illuminated layer next to the wall of the tube in order to pick up light energy. Turbulence would permit the cells to pick up light in this way and, in work to be reported elsewhere, we have found that the growth yield dropped dramatically if the Reynolds number in the tube fell below 1000 , that is, below the minimum for turbulent flow. Calculations of the mean time taken for cells to cycle from one side of a tube to the other in turbulent flow have been made by A. P. Ippen, quoted by Arthur D. Little Inc. (1953). These data suggest that, in our loop reactor with tubing of $0.7 \mathrm{~cm}$ bore, the mean cycle time would be of the order $0.5 \mathrm{~s}$. If the cycle time became of the order of $40 \mathrm{~s}$, through low turbulence or increase in the reactor depth, then the intermittent illumination effects reported here, that is, increase in maintenance energy and consequent reduction of yield, could occur even in the illuminated section. Lack of attention to this effect could lead to large discrepancies between the growth yields found in different laboratories using different types and sizes of photoreactor with varying degrees of turbulence. For example, in the pilot plant of Mituya et al. (1953) the dark fraction (sump plus tower) was 0.6 and the cycle time was 2 min. Such a large dark fraction, our model predicts, will cause a large endogenous metabolism (maintenance) effect and result in a substantial drop in the efficiency of biomass production.

A grant in aid of this research from Shell Research Ltd in gratefully acknowledged.

\section{REFERENCES}

Arthur D. Little InC. (1953). Pilot-plant studies in the production of Chlorella. In Algal Culture, pp. 235-272. Edited by J. S. Burlew. Washington, D.C.: Carnegie Institution of Washington.

Brooks, J. D. \& MEERs, J. L. (1973). The effect of discontinuous methanol addition on the growth of a carbon-limited culture of Pseudomonas. Journal of General Microbiology 77, 513-519.

Brown, R. C. (1961). A Textbook of Physics. London: Longman.

KoK, B. (1953). Experiments on photosynthesis by Chlorella in flashing light. In Algal Culture, pp.
63-75. Edited by J. S. Burlew. Washington, D.C.: Carnegie Institution of Washington.

LIVANSKÝ, K. (1979). Effect of the nonilluminated part of suspension on biomass production in an algal reactor. Folia microbiologica 24, 339-345.

Mituya, A., Nyunoya, T. \& Tamiya, H. (1953). Pre-pilot-plant experiments on algal mass culture. In Algal Culture, pp. 273-284. Edited by J. S. Burlew. Washington, D.C.: Carnegie Institution of Washington.

Neijssel, O. M. \& Tempest, D. W. (1976). Bioenergetic aspect of aerobic growth of Klebsiella 
aerogenes NCTC 418 in carbon-limited and carbonsufficient chemostat culture. Archives of Microbiology 107, 215-221.

Phillips, J. N., JR \& MYers, J. (1954). Growth rate of Chlorella in flashing light. Plant Physiology 29, 148-152.

PIRT, S. J. (1975). Principles of Microbe and Cell Cultivation. Oxford: Blackwell Scientific Publications.
PiRT, S. J., Panikov, N. \& Lee, Y.-K. (1979). The miniloop: a small scale air-lift microbial culture vessel and photobiological reactor. Journal of Chemical Technology and Biotechnology 29, 437441.

Pirt, S. J., Lee, Y.-K., Richmond, A. \& Watts PiRT, M. (1980). The photosynthetic efficiency of Chlorella biomass growth with reference to solar energy utilisation. Journal of Chemical Technology and Biotechnology 30, 25-34. 